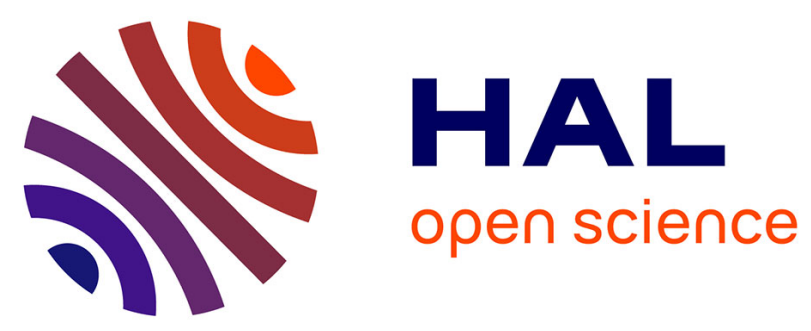

\title{
Multicoalitional solutions
}

Stéphane Gonzalez, Michel Grabisch

\section{To cite this version:}

Stéphane Gonzalez, Michel Grabisch. Multicoalitional solutions. Journal of Mathematical Economics, 2016, 64, pp. 1-10. halshs-01293785

\section{HAL Id: halshs-01293785 https://shs.hal.science/halshs-01293785}

Submitted on 28 Feb 2017

HAL is a multi-disciplinary open access archive for the deposit and dissemination of scientific research documents, whether they are published or not. The documents may come from teaching and research institutions in France or abroad, or from public or private research centers.
L'archive ouverte pluridisciplinaire HAL, est destinée au dépôt et à la diffusion de documents scientifiques de niveau recherche, publiés ou non, émanant des établissements d'enseignement et de recherche français ou étrangers, des laboratoires publics ou privés. 


\title{
Multicoalitional solutions *
}

\author{
Stéphane GONZALEZ ${ }^{\dagger}$ and Michel GRABISCH ${ }^{\ddagger}$ \\ email: stephane.gonzalez@univ-st-etienne.fr,michel.grabisch@univ-paris1.fr
}

Version of February 8, 2016

\begin{abstract}
The paper proposes a new concept of solution for TU games, called multicoalitional solution, which makes sense in the context of production games, that is, where $v(S)$ is the production or income per unit of time. By contrast to classical solutions where elements of the solution are payoff vectors, multicoalitional solutions give in addition an allocation time to each coalition, which permits to realize the payoff vector. We give two instances of such solutions, called the d-multicoalitional core and the c-multicoalitional core, and both arise as the strong Nash equilibria of two strategic games, where in the first utility per active unit of time is maximized, while in the second it is the utility per total unit of time. We show that the d-core (or aspiration core) of Benett, and the c-core of Guesnerie and Oddou are strongly related to the d-multicoalitional and c-multicoalitional cores, respectively, and that the latter ones can be seen as an implementation of the former ones in a noncooperative framework.
\end{abstract}

Keywords: cooperative game, core, aspiration core, strong Nash implementation JEL Classification: C71, C72

\section{Introduction}

Finding the solution of a cooperative game with transferable utility, that is, how to share among the players the benefit of cooperation (what we call here a distribution), is a well-known problem in game theory which has been studied in depth from a long time ago. One of the most prominent solutions is the core (Gillies, 1953, 1959), which is the set of efficient and coalitionally rational distributions (i.e., the payoff given to a coalition is at least equal to what the coalition could have achieved by itself; for applications of the core in economy, see, e.g., Trockel (2005); Shitovitz (1997); Flam and Koutsougeras (2010)).

Although quite attractive on a rational point of view since it ensures stability of the solution, the core does not contain any allocation in many cases. It is however possible to extend the definition of the core, so as to keep coalitional rationality while ensuring its nonemptiness. The $k$-additive core, proposed by Grabisch and Miranda (2008) and later developed by the authors (Gonzalez and Grabisch, 2015), achieves this goal by allowing distributions to be defined not only on individual players but also on coalitions up to a size $k$. The aim of this paper is

\footnotetext{
${ }^{*}$ We wish to thank Walter Trockel, Peter Sudhölter, Juan Camilo Gómez and an anonymous referee for their helpful comments.

${ }^{\dagger}$ Corresponding author. Université Paris 1, 106-112, Bd de l'Hôpital, 75013 Paris, France; Université de Lyon, Lyon, F-69007, France; CNRS, GATE Lyon Saint-Etienne, Ecully, F-69130, France; University Jean Monnet, Saint-Etienne, F-42000, France

${ }^{\ddagger}$ Paris School of Economics; Université Paris 1, 106-112, Bd de l’Hôpital, 75013 Paris, France
} 
to propose another way to generalize the core, by focusing on linear production games and introducing time into the picture.

We consider a linear production game $v$, that is, for any coalition of players $S, v(S)$ represents the production per unit of time of the coalition $S$, or more directly, the income per unit of time, which has to be redistributed among the players. The classical notion of core is based on the simplistic assumption that the best arrangement of the players (in terms of maximizing the income) is to put them all together, in other words, the grand coalition $N$ forms and $v(N)$ is the income per unit of time to be redistributed. Alternatively, the c-core (Guesnerie and Oddou, 1979; Sun et al., 2008), or coalition structure core (Kóczy and Lauwers, 2004) supposes that the grand coalition is not necessarily the best arrangement, but considers every possible partition of the grand coalition and takes the partition which achieves the maximum.

Yet this generalization is not powerful enough since the c-core is sometimes empty. Generalizing partitions to balanced collections permits to ensure nonemptiness in any case, and this gives rise to the d-core (Albers, 1979), or aspiration core (Bennett, 1983; Cross, 1967).

Let us elaborate on balanced collections and their interpretation. It is common in the literature to see a balancing weight of a coalition in a balanced collection as the fraction of time this coalition is active (see, e.g.,Peleg and Sudhölter (2003)). Then it is usually considered that a coalition being active during a given fraction of time receives the corresponding fraction of its income (Aumann, 1989). With this view in mind, a feasible payoff corresponds to the maximum income players can generate if each of them devotes one unit of time among the coalitions to which they belong: This set is equal to the set of aspiration feasible payoffs. Then the theorem of Bondareva-Shapley (Bondareva, 1963; Shapley, 1967) ensures that there exists a way to share time among coalitions, which builds payoff satisfying coalitional rationality. Thus, the aspiration core seems to be a suitable way to describe how coalitions must form if each player has one unit of time, and how long each coalition must be active.

However, the d-core does not explain which coalitions form and in which time frame. Cross (1967) informally describes the d-core as a set of stable coalitions with their associated payoffs. The $\mathcal{B}$-core and the $\mathcal{M}$-core proposed by Cesco (2012) are closely related to or constitute a continuation of the paper of Cross, by considering the set of coalitions which lead to a payoff into the d-core. Under this view, a cooperative TU-game solution should be not only composed of the payoffs given to each player but should also comprise the time alloted to each coalition which permits to achieve these payoffs.

A second major concern is that the "value" or "utility" of time is ignored. Even if each player is active during exactly one unit of time, under the natural assumption that a player cannot be active in two different coalitions at the same time, the implementation of the solution may require a total amount of time greater than one unit: Imagine a situation, like the one described by the game below, with three players where reaching the payoff given by the d-core needs an allocation equals to one half unit of time for every pair of players ${ }^{1}$.It follows that any implementation of this situation needs a minimum of 1.5 units of time. The question is then: is the player concerned with only the time he is active, or by the total duration of the process? In the former case, since the time of activity of each player is 1 by definition, this amounts to consider that players are concerned with the income per unit of time. Again, the d-core lacks precision in describing a solution.

In order to overcome these drawbacks, we propose a completely different approach to the problem, having its root in noncooperative game theory: We suppose that each player proposes the formation of a coalition for a chosen amount of time and claims a payoff for his participation

\footnotetext{
${ }^{1}$ Such a situation is described with the island desert story in the introduction of Garratt and Qin (2000).
} 
in each coalition which is formed ${ }^{2}$, each of these proposals being seen as a strategy. Moreover, a utility function is defined over the set of strategies. The notion of Strong Nash equilibrium (Aumann, 1959) seems to be the adequate notion here, since it ensures stability of any coalition by preventing any coalitional deviation. Then, a solution in our framework is precisely the set of undominated strategies (in the sense of strong Nash equilibrium). We emphasize the fact that, in our framework, each element of the solution is a pair $(x, \alpha)$, where $x$ is a payoff vector, and $\alpha$ is a time allocation for every coalition. This constitutes to our opinion an innovation since, up to our knowledge, no former work explicitely proposes a solution under this form. We call multicoalitional solution such kind of solution.

We propose two different types of utility functions, leading to two kinds of strong Nash equilibria. The first one is the utility per active unit of time, and leads to the maximization of the hourly wage. We call d-multicoalitional core the set of such equilibria, and we show that this set is never empty, and that its elements satisfy nonnegativity, coalitional rationality and a notion of efficiency close to the one of the d-core (Theorem 3). We show the exact relation between the d-core and the d-multicoalitional core: in short, vectors of utility of strategies in the d-multicoalitional core are elements of the d-core (Proposition 6) when each player play the same amount of time. The second type of utility function is the utility per total unit of time, and leads to the maximization of the total income. We prove that any strong Nash equilibrium of this type can be turned into a strategy which is also a strong Nash equilibrium of the first type (Proposition 8). Therefore, we define the c-multicoalitional core as the set of strategies which are strong Nash equilibria for both problems. They are nonempty as soon as the c-core is not empty, moreover, a relation between the c-core and the c-multicoalitional core is established (Theorem 4).

The paper is organized as follows. Section 1 introduces the basic definitions and notation. Section 2 introduces time allocations for coalitions and timetables, that is, how to organize coalition formation so that no conflict occurs, as well as the notion of minimal duration for timetables. Section 3 presents the model with a utility corresponding to a hourly wage and proves the existence of strong Nash equilibria defining the d-multicoalitional core which we characterize. Section 4 gives a simple motivating example proving that our framework is expendable to games with unfeasible coalitions. Section 5 studies the existence of strong Nash equilibria with a utility corresponding to a utility per hour lived and define the c-multicoalitional core which we characterize.

\section{Notation and basic concepts}

Let $N$ denote a fixed finite nonempty set with $n$ members, who will be called agents or players. Coalitions of players are nonempty subsets of $N$, denoted by capital letters $S, T$, and so on. Whenever possible, we will omit braces for singletons and pairs, denoting $\{i\},\{i, j\}$ by $i, i j$ respectively, in order to avoid a heavy notation. We denote by $\Pi(N)$ the set of partitions of $N$. A transferable utility (TU) game on $N$ is a pair $(N, v)$ where $v$ is a mapping $v: 2^{N} \rightarrow \mathbb{R}$ satisfying $v(\emptyset)=0$. We will denote by $\mathcal{G}(N)$ the set of mappings $v$ over $N$ such that $(N, v)$ is a TU game. For any coalition $S, v(S)$ represents the worth of $S$, i.e., what coalition $S$ could earn regardless of other players. A payoff vector is a vector $x \in \mathbb{R}^{n}$ that assigns to agent $i$ the payoff $x_{i}$. For any coalition $T \subset N$, we denote by $v_{T}$ the restriction of $v$ to $2^{T}$. Given $x \in \mathbb{R}^{n}$, and $S \subseteq N$, denote by $x(S)$ the sum $\sum_{i \in S} x_{i}$ with the convention that $x(\emptyset)=0$. A nonempty

\footnotetext{
${ }^{2} \mathrm{~A}$ similar game is proposed by Bejan and Gómez (2012a). In their paper, agents have to share one unit of time among the set of coalitions to which they belong. The game leads to a strong Nash implementation of the d-core (see Proposition 6).
} 
collection $\mathcal{B} \subseteq 2^{N}$ is called balanced (over $N$ ) if positive numbers $\delta_{S}, S \in \mathcal{B}$, exist such that :

$$
\sum_{S \in \mathcal{B}} \delta_{S} \chi^{S}=\chi^{N}
$$

where $\chi^{S}$ is the characteristic vector of $S$ given by $\chi_{i}^{S}=1$ if $i \in S$ and 0 otherwise. The collection $\left(\delta_{S}\right)_{S \in \mathcal{B}}$ is called a system of balancing weights. We say that $\left(\mathcal{B},\left(\delta_{S}\right)_{S \in \mathcal{B}}\right)$ is a balanced system if $\mathcal{B}$ is balanced and $\left(\delta_{S}\right)_{S \in \mathcal{B}}$ is a corresponding system of balancing weights. A balanced collection is minimal if no subcollection of it is balanced. It is well known that a balanced collection is minimal if and only if there is a unique system of balancing weights.

Let $v \in \mathcal{G}(N)$. An allocation is said to be coalitionally rational if for each coalition $S$ we have $x(S) \geq v(S)$. A core-solution collects coalitionally rational allocations that meet a feasibility condition. Different feasibility conditions define different core-solutions: the core (resp., c-core, and the d-core) collects those coalitionally rational allocations that satisfy $x(N)=v(N)$ (resp.,

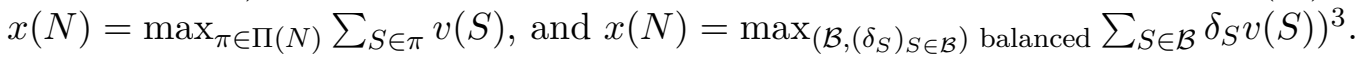

In the classical view of core, it is supposed that the grand coalition will form, and its worth is shared among the players. With the c-core, the best partition of the players is sought, in order to maximize the total worth. Now, the d-core generalizes the c-core since partitions are particular balanced collections. More importantly, the d-core has an interesting interpretation if one considers $\delta_{S}$ for $S \in \mathcal{B}$ as an amount of time ${ }^{4}$ allocated to $S$. Assuming that $v(S)$ represents the worth or the production per unit of time, $\delta_{S} v(S)$ is the total worth/production achieved by $S$. Under this viewpoint, an allocation of the d-core is a coalitionally rational sharing among the players of the total worth which can be achieved by the best arrangement of the players in time.

We say that a game $v$ is balanced if $\mathrm{C}(v)$ is nonempty, and c-balanced if $\mathrm{c}-\mathrm{C}(v)$ is nonempty. It is well known and easy to prove that for any game $v$ in $\mathcal{G}(N)$, we have:

$$
\mathrm{C}(v) \subseteq \mathrm{c}-\mathrm{C}(v) \subseteq \mathrm{d}-\mathrm{C}(v),
$$

and equality holds everywhere if and only if $v$ is balanced. ${ }^{5}$

Example 1. Let us consider a simple numerical example ${ }^{6}$ with three agents who can work in the way they want, i.e., they can freely form coalitions during some time decided by them. We suppose that the productivity depends on the coalition which is formed (or active). Furthermore, any coalition which decides to be active has determined beforehand, by contract, the sharing that their members will receive. The following table gives for each possible coalition its production rate per hour:

\begin{tabular}{|c|ccccccc|}
\hline Coalition $S$ & 1 & 2 & 3 & 12 & 13 & 23 & 123 \\
\hline Productivity of the coalition $v(S)$ & 0 & 0 & -4 & 5 & 0 & 0 & 2 \\
\hline
\end{tabular}

If agents 1 and 2 are working one hour together, they can share an amount of 5 , and if agent 3 works alone during one hour, he must pay $6^{7}$, while the grand coalition receives an amount of 2 per hour. Standard solution concepts of cooperative game theory, e.g., the core, typically

\footnotetext{
${ }^{3}$ The concept of core (resp. c-core, and d-core) is due to Gillies (1953) (resp. Guesnerie and Oddou (1979), and Bennett (1983); Albers (1974); Turbay (1977)).

${ }^{4}$ Although usually it is interpreted as an amount of resources (Kannai, 1992).

${ }^{5}$ The inclusions where shown by, e.g., Bejan and Gómez (2012b), and the last equality by Cross (1967) and Bennett (1983).

${ }^{6} \mathrm{~A}$ more elaborate example can be found in section 4.

${ }^{7}$ Observe that the usual definition of a cooperative game allows nonpositive worths. To fix the ideas, one can imagine a situation where the cost of forming or activing a coalition is larger than its benefit.
} 
consider that the grand coalition (or a partition of it) is already formed and that a sharing acceptable for everyone must be found according to some rationality principles. In our example, the grand coalition is the partition which can create the best worth. However, the core of this game is empty, that is, for any sharing of 2 , one can find a blocking coalition: if we denote by $x_{i}$ the payoff for the player $i$ coming from a sharing of 2 , that is if $x_{1}+x_{2}+x_{3}=2$, then according to the Bondareva-Shapley theorem (Bondareva, 1963; Shapley, 1967), it is impossible to ensure $\sum_{i \in S} x_{i} \geq v(S)$ for every coalition $S$. A second possibility, using the d-core (Cross, 1967), consists in considering that each coalition 12, 13 and 23 works 30 minutes. This coalitional process creates an output equal to 2.5 and it becomes possible to find a payoff $x_{1}=x_{2}=2.5$ and $x_{3}=-2.5$ which satisfies $x_{1}+x_{2}+x_{3}=2.5$ and $\sum_{i \in S} x_{i} \geq v(S)$ for every coalition $S$. A contract which ensures this payoff to each player in return of a time activity of 30 minutes per pair of players seems to be acceptable. However, this solution has two drawbacks:

(i) Player 3 has to pay something, and it seems quite unreasonable for him to accept to work for a negative wage.

(ii) Coalition 12 receives a "rational payoff" according to its activity but, the coalition formation lasting $1 \mathrm{~h} 30$, if the utility of the payment is perceived relatively to the total duration, coalition 12 can block the payoff since it can receive by itself the same amount by working only one unit of time in total.

Hence, given that players are free to form coalitions and work or not, it seems more reasonable for player 3 not to work at all, while players 1 and 2 work for example one hour together. The corresponding payoff becomes $x_{1}=x_{2}=2.5$ and $x_{3}=0$. This payoff cannot be blocked by any coalition even if we consider that the worth of a coalition is discounted when its formation is delayed longer than one unit of time.

\section{Time allocation and optimal timetable}

A time allocation for coalitions in $N$ is a function $\alpha: 2^{N} \rightarrow[0, \infty[$. The collection $\mathcal{A}=\{S \in$ $\left.2^{N} \mid \alpha(S)>0\right\}$ is the collection of active coalitions under $\alpha$. The time allocation is in standard form if $\left(\mathcal{A},(\alpha(S))_{S \in \mathcal{A}}\right)$ is a balanced system. $\alpha(S)$ can be interpreted as the initial endowment of unit of time for coalition $S \in \mathcal{A}$. We denote by $\mathcal{T}(N)$ the set of time allocations in $N$.

We define a multicoalitional payoff vector as a pair $(x, \alpha)$, where $x \in \mathbb{R}^{N}$ is a payoff vector, and $\alpha \in \mathcal{T}(N)$ is a time allocation. A multicoalitional solution is a mapping which assigns to every game $(N, v)$ a set of multicoalitional payoff vectors.

Given a time allocation $\alpha$, it remains to specify how each active coalition under $\alpha$ will spend its allocated time, by means of a "timetable": at each time $t$, the timetable indicates which coalitions are active under $\alpha$. This timetable should satisfy two consistency requirements:

(i) locational consistency: A player cannot be in two different coalitions at the same time.

(ii) time consistency: the total amount of time spent by coalition $S$ should be equal to its time allocation $\alpha(S)$.

We also add two requirements in order to avoid bizarre or obviously inefficient timetables. First, for each coalition, the active period should be a union of intervals of positive duration (isolated instants are not allowed). Second, the timetable should not contain "holes", that is, periods during which no coalition is active. These considerations lead to the following formal definition: 
Let $\alpha$ be a time allocation, and $\mathcal{A}$ its collection of active coalitions. We call coalition timetable a function $f_{\alpha}:\left[0,+\infty\left[\rightarrow 2^{\mathcal{A}}\right.\right.$ which satisfies the following properties:

(i) for each $t \in\left[0,+\infty\left[\right.\right.$, and each pair $S, T$ in $f_{\alpha}(t)$, we have $S=T$ or $S \cap T=\emptyset$;

(ii) For any coalition $S$ in $\mathcal{A}, \tau(S)=\left\{t \in\left[0,+\infty\left[\mid f_{\alpha}(t) \ni S\right\}\right.\right.$ is a union of disjoint intervals of positive Lebesgue measure, and in addition, $l(\tau(S))=\alpha(S)$, where $l$ is the Lebesgue measure on $\mathbb{R}$;

(iii) If $f_{\alpha}(t)=\emptyset$ for some $t \in\left[0,+\infty\left[\right.\right.$, then $f_{\alpha}\left(t^{\prime}\right)=\emptyset$ for all $t^{\prime}>t$.

Note that because of (iii), every timetable starts at $t=0$. We denote by $\mathcal{F}(\alpha)$ the set of all timetables for time allocation $\alpha$. Note that this set is in general not denumerable. Indeed, take $n=3$ and coalitions 12, 23 with alloted time 1 to each of them. Then $f(t)=12$ for $t \in[a, a+1]$ and $f(t)=23$ for $t \in[0, a[\cup] a+1,2]$ is a possible timetable for any $a \in] 0,1[$.

The duration of a timetable $f_{\alpha}$ is defined as

$$
d_{\max }\left(f_{\alpha}\right)=\sup \left\{t \in \left[0,+\infty\left[\mid f_{\alpha}(t) \neq \emptyset\right\} .\right.\right.
$$

Due to conditions (ii) and (iii) in the definition of a timetable, $d_{\max }\left(f_{\alpha}\right)$ is bounded above by $\sum_{S \in \mathcal{A}} \alpha(S)<\infty$, therefore $d_{\max }\left(f_{\alpha}\right)$ is finite. For a time allocation $\alpha$, the minimal duration of $\alpha$ is

$$
d(\alpha)=\inf \left\{d_{\max }\left(f_{\alpha}\right), f_{\alpha} \in \mathcal{F}(\alpha)\right\} .
$$

Remark 1. Since the map $d$ is homogenous - the equality $d(r \alpha)=r d(\alpha)$ holds for each $r \in \mathbb{R}^{+}$ and for each time allocation $\alpha$ - the map $d$ is ratio scale invariant. The result, thus, does not depend upon the unit of time measurement.

Note that $d(\alpha)$ is finite since each duration is a positive finite number.

Theorem 1. For each time allocation $\alpha$, there exists a timetable $f_{\alpha}^{*}$ with a duration $d(\alpha)$.

Proof. We denote by $\mathcal{F}(\alpha)$ the set of coalition timetables associated with time allocation $\alpha$. Let $\mathcal{T}$ be the product topology on $\mathcal{F}(\alpha)$. We know by Tychonoff theorem that $\mathcal{F}(\alpha)$ is compact with the topology $\mathcal{T}$. Let $\left(f_{n}\right)_{n \in \mathbb{N}}$ in $\mathcal{F}(\alpha)^{\mathbb{N}}$ be a sequence which converges to some $f \in \mathcal{F}(\alpha)$. Since $\mathcal{F}(\alpha)$ is equipped with the product topology, we have:

$$
\forall t \in\left[0,+\infty\left[, \lim _{n \rightarrow+\infty} f_{n}(t)=f(t) .\right.\right.
$$

Since $\mathcal{F}(\alpha)$ is a space of functions valued in a finite set, we have:

$$
\forall t \in\left[0,+\infty\left[, \exists N_{t} \in \mathbb{N}, \forall n \geq N_{t}, f_{n}(t)=f(t) .\right.\right.
$$

Hence,

$$
\exists N_{d_{\max }(f)} \in \mathbb{N}, \forall n \geq N_{d_{\max }(f)}, f_{n}\left(d_{\max }(f)\right)=f\left(d_{\max }(f)\right) \neq \emptyset,
$$

and we deduce that:

$$
\inf _{k \geq N_{d_{\max }(f)}} d_{\max }\left(f_{k}\right) \geq d_{\max }(f)
$$

Therefore,

$$
d_{\max }(f) \leq \liminf _{n \rightarrow+\infty} d_{\max }\left(f_{n}\right) .
$$

On the other hand, the definition of inf implies that for all $j \in \mathbb{N}$, there exists $f_{j} \in \mathcal{F}(\alpha)$ such that $d_{\max }\left(f_{j}\right)-d(\alpha) \leq \frac{1}{j}$. Hence, $\lim _{j \rightarrow+\infty} d_{\max }\left(f_{j}\right) \rightarrow d(\alpha) \in[0,+\infty[$. Since $\mathcal{F}(\alpha)$ is compact, 
there exists a subsequence $\left(f_{j_{k}}\right)_{k \in \mathbb{N}}$ of $\left(f_{j}\right)_{j \in \mathbb{N}}$ and some $f \in \mathcal{F}(\alpha)$ so that $\lim _{k \rightarrow+\infty} f_{j_{k}}=f$. Since the limit $\lim _{j \rightarrow+\infty} d_{\max }\left(f_{j}\right)$ exists and is equal to $d(\alpha)$, it follows that $\lim _{k \rightarrow+\infty} d_{\max }\left(f_{j_{k}}\right)$ exists and is equal to $d(\alpha)$

Therefore, by (1),

$$
d_{\max }(f) \leq \liminf _{k \rightarrow+\infty} d_{\max }\left(f_{j_{k}}\right)=\lim _{k \rightarrow+\infty} d_{\max }\left(f_{j_{k}}\right)=d(\alpha)=\inf \left\{d_{\max }\left(f_{\alpha}\right), f_{\alpha} \in \mathcal{F}(\alpha)\right\}
$$

Hence

$$
d_{\max }(f)=d(\alpha)
$$

Any timetable with duration $d(\alpha)$ is called an optimal timetable of $\alpha$.

Example 2. Let $N=\{1,2,3,4,5\}$ and consider the following collection $\mathcal{A}$ with its time allocation:

\begin{tabular}{|c|ccccc|}
\hline$S$ & 12 & 23 & 34 & 45 & 15 \\
\hline$\alpha(S)$ & $1 / 2$ & $1 / 2$ & $1 / 2$ & $1 / 2$ & $1 / 2$ \\
\hline
\end{tabular}

Note that this is a minimal balanced system. We give two examples of timetable in Figure 1.
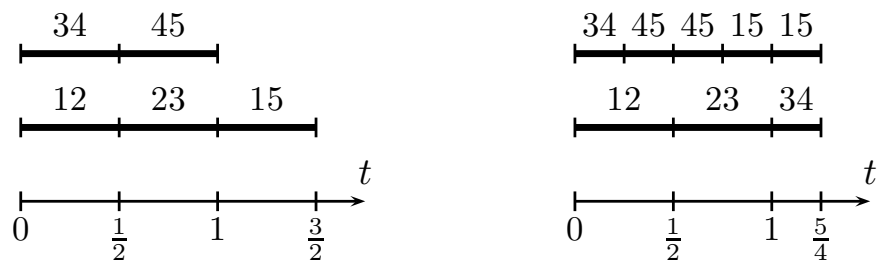

Figure 1: Time allocation for a 5-player game: Two examples of timetable

The problem of computing the minimal duration in general seems to be extremely complex. We give hereafter a lower and an upper bound of it. We recall that in a graph $\mathfrak{G}=(V, E)$ with set of vertices $V$ and set of edges $E$, a clique is a subset of vertices which are pairwise connected (by an edge) in $\mathfrak{G}$. An independent set is a subset of vertices $I$ such that no pair of vertices is connected in $\mathfrak{G}$. A partition $\mathcal{K}=\left\{I_{1}, \ldots, I_{m}\right\}$ of $V$ in independent sets is called a coloring of $\mathfrak{G}$. Given a time allocation $\alpha$ in $N$, we construct the graph $\mathfrak{G}=(\mathcal{A}, E)$, where the set of vertices is the set of active coalitions under $\alpha$, and there is an edge $(S, T)$ if and only if $S \cap T \neq \emptyset$.

Theorem 2. For any time allocation $\alpha$ in $N$, the minimal duration satisfies

$$
\max _{\mathcal{C}: \text { clique of } \mathfrak{G}} \alpha(\mathcal{C}) \leq d(\alpha) \leq \min _{\mathcal{K}: \text { coloring of } \mathfrak{G}} \alpha(\mathcal{K}),
$$

with $\alpha(\mathcal{C})=\sum_{S \in \mathcal{C}} \alpha(S)$, and $\alpha(\mathcal{K})=\sum_{I \in \mathcal{K}} \max _{S \in I} \alpha(S)$.

Proof. By condition (i) in the definition of a timetable, the subsets in a clique $\mathcal{C}$ must have disjoint time slots in a timetable. This being true for any clique leads to the lower bound.

Now, observe that if no splitting of time slots is allowed, (i.e., if $\tau(S)$ is a single interval for all $S \in \mathcal{C}$ ), finding a timetable amounts to finding a coloring of the graph, and its duration is the sum of maximal (in term of $\alpha(S)$ ) coalitions in each independent set. Taking a coloring minimizing this duration gives the upper bound, since splitting of time slots can only improve this solution. 
The next result concerns time allocations in standard form.

Proposition 1. Let $\alpha$ be a time allocation in standard form. Then $d(\alpha) \geq 1$, and a timetable $f_{\alpha}$ satisfies $d_{\max }\left(f_{\alpha}\right)=1$ if and only if for each $t \in[0,1], f_{\alpha}(t)$ is a partition of $N$, and is $\emptyset$ otherwise.

Proof. For each $i \in N$, the collection $\mathcal{A}(i)=\{S \in \mathcal{A} \mid S \ni i\}$ is a clique of $\mathfrak{G}$, and $\alpha(\mathcal{A}(i))=1$ since $\mathcal{A}$ is a balanced collection. It follows from Theorem 2 that $d(\alpha) \geq 1$.

Take $f_{\alpha}$ such that $d_{\max }\left(f_{\alpha}\right)=1$, and suppose that there exists a nonnull (in the sense of Lebesgue measure) time interval in $[0,1]$ on which $f_{\alpha}$ is not a partition of $N$. This would mean that there exists some $i \in N$ such that on a nonnull time interval $\Delta \subseteq[0,1], \bigcup_{S \in f_{\alpha}(t)} S$ does not contain $i$ for all $t \in \Delta$. However, since $\alpha$ is in standard form, we have $\alpha(\mathcal{A}(i))=1$, which would imply that for some nonnull interval $\left.\Delta^{\prime} \subseteq\right] 1, \infty\left[, f_{\alpha}(t) \ni S \ni i\right.$ for all $t \in \Delta^{\prime}$, a contradiction with $d_{\max }(\alpha)=1$. The converse is obvious.

Note that if $f_{\alpha}$ has the above property, it does not necessarily imply that the set of active coalitions under $\alpha$ is a collection of partitions, as the next example shows: Consider $N=$ $\{1,2,3\}$, and a timetable $f_{\alpha}$ defined on $[0,1]$ as follows: $f_{\alpha}(t)=\{12,3\}$ for $t \in\left[0, \frac{1}{3}\right], f_{\alpha}(t)=$ $\{1,2,3\}$ for $\left.t \in] \frac{1}{3}, \frac{2}{3}\right]$, and $f_{\alpha}(t)=\{13,2\}$ for $\left.\left.t \in\right] \frac{2}{3}, 1\right]$. Then $\mathcal{A}=\{1,2,3,12,13\}$, which is not a collection of partitions.

We say that a timetable $f_{\alpha}$ has $m$ full rows of identical length if for every $t$ such that $f_{\alpha}(t) \neq \emptyset,\left|f_{\alpha}(t)\right|=m$. A simple sufficient condition for optimality is the following.

Proposition 2. Let $\alpha$ be a time allocation, $\mathcal{A}$ its collection of active coalitions, and $m$ be the size of a greatest independent set (i.e., one can find at most $m$ disjoint subsets in $\mathcal{A}$ ). A timetable $f_{\alpha}$ is optimal if it has $m$ full rows of identical length. Consequently, a lower bound for $d(\alpha)$ is

$$
d(\alpha) \geq \frac{1}{m} \sum_{S \in \mathcal{A}} \alpha(S)
$$

Proof. If $f_{\alpha}$ were not optimal, it would be possible to shorten its duration by taking the sets being active on some interval $\left[t_{0}, t_{1}\right]$ and dispatching them on other time intervals. However, for every $t$ in the domain of $f$, it is not possible to add a set to $f_{\alpha}(t)$ because there is no independent set of size greater than $m$. The statement on the lower bound directly follows.

Coming back to Example 2, the corresponding graph is a cycle of length 5 , therefore, maximal cliques are for example $\{12,23\}$, which gives a lower bound of 1 . On Figure 1, the left timetable implements the upper bound solution (no splitting), while the right figure is the optimal solution. Indeed, it has two full rows of identical length, and there is at most two disjoint sets in $\mathcal{A}$. Therefore the optimal duration $d(\alpha)$ is $\frac{5}{4}$. This shows that time allocation in standard form does not have necessarily an optimal duration equal to 1 (see Proposition 1).

\section{Strategic coalition formation}

\subsection{The model}

We denote by $v$ a function that associates with each subset $S$ of $N$, a real number, $v(S)$ which represents the payoff that the coalition $S$ could create alone during one unit of time. We assume that a coalition $S$ which is active during $\alpha(S)$ unit of time produces a payoff equals to $\alpha(S) v(S)$. 
Then to each time allocation $\alpha$, we can associate the total payoff $G(v, \alpha)$ produced during at least $d(\alpha)$ units of time and defined by:

$$
G(v, \alpha):=\sum_{S \subseteq N} \alpha(S) v(S) .
$$

We say that a payoff vector $z$ is $(v, \alpha)$-feasible if it satisfies $z(N) \leq G(v, \alpha)$. We assume that each player $i \in N$ chooses a strategic time allocation $\alpha_{i} \in \mathbb{R}^{2^{N}}$ that associates with each subset $S$ of $N$ a positive real number $\alpha_{i}(S)$ which represents the amount of unit of time that the player $i$ wants to spend in coalition $S$. We suppose that $\alpha_{i}(S)=0$ if $S \not \ngtr i$, that is, $i$ cannot devote any time in a coalition to which he does not belong. We denote by $\mathcal{T}_{i}(N) \subseteq \mathcal{T}(N)$ the set of strategic time allocations of the player $i \in N$. We assume that the length of activity period $\bar{\alpha}(S)$ of a coalition $S$ is equal to the minimum amount of time that each member wants to spend inside: The first who wants to leave a coalition decides the lifetime of this coalition. Hence, for a vector $\left(\alpha_{i}\right)_{i \in N} \in \prod_{i \in N} \mathcal{T}_{i}$ of strategic time allocations, we can associate a time allocation called lifetime and denoted by $\bar{\alpha}$ defined by:

$$
\bar{\alpha}(S)=\min _{i \in S} \alpha_{i}(S) .
$$

The lifetime of a coalition means the following: a coalition $S$ is active only in case each player $i$ in $S$ is willing to activate coalition $S$. As a consequence, the length of period during which $S$ is active is equal to the minimum of the $\alpha_{i}(S)$ with $i$ in $S$.

A strategy of a player $i \in N$ is a pair $\left(x_{i}, \alpha_{i}\right) \in \mathbb{R} \times \mathcal{T}_{i}(N)$, where $x_{i}$ represents the payoff that the player $i$ wants to have for his participation, and $\alpha_{i}$ is a strategic time allocation of the player $i$. We consider two kinds of utilities: The first one puts to the fore the desire for each agent to maximize his payoff with regard to the length of his active participation, that is, the desire to have the best "hourly wage", while the second utility gives emphasis to the desire of agent for maximising his payoff at the end of the timetable or equivalently to maximize his "compensation per hour lived". Since time for forming coalition is real and the payoff received at the end of the coalition formation process can be seen as a contract payment, it is natural to discount the worth of a coalition when players have to wait more than one unit of time for receiving their payoff. The second utility clearly reflects discounting. Moreover, we suppose that players who propose an allocation which is non "feasible" receive a punishment equal to -1 , similarly to Nash's demand game. We can also restrict the space of strategies and forbid the non feasible allocations without deeply change the results which will follow. We denote by $u_{i}$ and we call utility per active unit of time of the player $i$ the function defined over $\mathbb{R}^{N} \times \prod_{j \in N} \mathcal{T}_{j}$ by:

$$
u_{i}\left(x,\left(\alpha_{j}\right)_{j \in N}\right):=\left\{\begin{array}{lc}
0 & \text { if } \sum_{S \ni i} \alpha_{i}(S)=0 \text { and } x_{i}=0 \\
\sum_{S \ni i} \alpha_{i}(S) & \text { if } x \text { is }(v, \bar{\alpha}) \text {-feasible and } \sum_{S \ni i} \alpha_{i}(S) \neq 0 \\
-1 & \text { otherwise. }
\end{array}\right.
$$

On the other hand, we denote by $\tilde{u}_{i}$ and we call utility per total unit of time of the player $i$ the function defined over $\mathbb{R}^{N} \times \prod_{j \in N} \mathcal{T}_{j}$ by:

$$
\tilde{u}_{i}\left(x,\left(\alpha_{j}\right)_{j \in N}\right):=\left\{\begin{array}{lc}
0 & \text { if } d(\bar{\alpha})=0 \\
\frac{x_{i}}{d(\bar{\alpha})} & \text { if } x \text { is }(v, \bar{\alpha}) \text {-feasible and } d(\bar{\alpha}) \neq 0 \\
-1 & \text { otherwise. }
\end{array}\right.
$$

Let $\Gamma=\left(N ; \Sigma_{1}, \cdots, \Sigma_{n} ; g_{1}, \cdots, g_{n}\right)$ be a $n$-player strategic game, where $\Sigma_{i}$ is the set of strategies of the player $i$, and the mapping $g_{i}: \prod_{j \in N} \Sigma_{j} \mapsto \mathbb{R}$ represents the utility of $i$. For 
$S \subseteq N$, we denote by $\Sigma^{S}$ the set $\Sigma^{S}:=\prod_{i \in S} \Sigma_{i}$, and for $\sigma \in \Sigma^{N}$, we denote by $\sigma_{S}$ the projection of $\sigma$ on $\Sigma^{S}$.

We say that $\sigma^{*} \in \Sigma^{N}$ is a strong Nash equilibrium of $\Gamma$, (Aumann, 1959) or strong equilibrium of $\Gamma$ if one has :

$$
\forall S \subseteq N, \forall \sigma_{S} \in \Sigma^{S}, \exists i \in S, g_{i}\left(\sigma^{*}\right) \geq g_{i}\left(\sigma_{S}, \sigma_{N \backslash S}^{*}\right)
$$

In the following two subsections, we define and study the strong Nash equilibria of $(N ; \mathbb{R} \times$ $\left.\mathcal{T}_{1}, \cdots, \mathbb{R} \times \mathcal{T}_{n} ; u_{1}, \cdots, u_{n}\right)$ and $\left(N ; \mathbb{R} \times \mathcal{T}_{1}, \cdots, \mathbb{R} \times \mathcal{T}_{n} ; \tilde{u}_{1}, \cdots, \tilde{u}_{n}\right)$.

\subsection{Strong Nash equilibria of $\left(N ; \mathbb{R} \times \mathcal{T}_{1}, \cdots, \mathbb{R} \times \mathcal{T}_{n} ; u_{1}, \cdots, u_{n}\right)$}

If we want to model a coalition formation, the minimal requirement is to enable coalitions to evolve as they want. Even if it rarely exists, the concept of strong Nash equilibrium seems to be a concepts which fits particularly well with this requirement since it allows for deviations by every coalition.

Let $v$ be a TU-game, we denote by $\mathrm{d}-M C(v)$ and we call $d$-multicoalitional core of $v$ the set of strong Nash equilibria of $\left(N ; \mathbb{R} \times \mathcal{T}_{1}, \cdots, \mathbb{R} \times \mathcal{T}_{n} ; u_{1}, \cdots, u_{n}\right)$.

We show that the d-multicoalitional core is never empty, and that it has properties close to the ones of the classical core, which justify its name.

Theorem 3. For any game $v$ in $\mathcal{G}(N)$, the set d- $M C(v)$ is nonempty, futhermore, $\left(x,\left(\alpha_{i}\right)_{i \in N}\right) \in$ $\mathrm{d}-M C(v)$ if and only if:

(i) $\forall i \in N, u_{i}\left(x,\left(\alpha_{i}\right)_{i \in N}\right) \geq 0$

(ii) $\forall S \subseteq N, \sum_{i \in S} u_{i}\left(x,\left(\alpha_{i}\right)_{i \in N}\right) \geq v(S)$ (coalitional rationality)

(iii) $x(N)=G(v, \bar{\alpha})((v, \bar{\alpha})$-efficiency)

Proof. Let $v$ be a game in $\mathcal{G}(N)$. First, we prove the nonemptiness property. Let $I$ be the subset of $N$ defined by $I:=\{i \in N, v(i)<0\}$. We define the game $\tilde{v}$ by $\tilde{v}(i)=0$ if $i \in I$ and $\tilde{v}(S)=v(S)$ otherwise. Let $x \in \mathrm{d}-\mathrm{C}(\tilde{v})$. There exists a balanced system $\left(\mathcal{A},\left(\lambda_{S}\right)_{S \subseteq N}\right)$ such that

$$
x(N)=\sum_{S \subseteq N} \lambda_{S} \tilde{v}(S) .
$$

We put $\alpha_{i}(i)=0$ if $i \in I$ and $\alpha_{i}(S)=\lambda_{S}$ otherwise. Hence:

$$
\sum_{S \subseteq N} \lambda_{S} \tilde{v}(S)=\sum_{S \subseteq N} \bar{\alpha}(S) v(S)=x(N)
$$

We want to prove that the strategy $\left(x,\left(\alpha_{i}\right)_{i \in N}\right)$ belongs to the d-multicoalitional core. Suppose there exists for a coalition $S \subseteq N$ a coalitional deviation $\left(\left(x_{i}^{\prime}\right)_{i \in S},\left(\alpha_{i}^{\prime}\right)_{i \in S}\right)$ such that $\forall i \in S$, $u_{i}\left(x,\left(\alpha_{i}\right)_{i \in N}\right)<u_{i}\left(\left(x_{i}\right)_{i \in N \backslash S},\left(x_{i}^{\prime}\right)_{i \in S},\left(\alpha_{i}\right)_{i \in N \backslash S},\left(\alpha_{i}^{\prime}\right)_{i \in S}\right)$. Since $\forall i \in N, 0 \leq u_{i}\left(x,\left(\alpha_{i}\right)_{i \in N}\right)$, the strict inequality $\forall i \in S, u_{i}\left(x,\left(\alpha_{i}\right)_{i \in N}\right)<u_{i}\left(\left(x_{i}\right)_{i \in N \backslash S},\left(x_{i}^{\prime}\right)_{i \in S},\left(\alpha_{i}\right)_{i \in N \backslash S},\left(\alpha_{i}^{\prime}\right)_{i \in S}\right)$ and the definition of $u_{i}$ ensure that the $\left(v,\left(\left(\alpha_{i}\right)_{i \in N \backslash S},\left(\alpha_{i}^{\prime}\right)_{i \in S}\right)\right)$-efficiency is satisfied. We denote by $\bar{\alpha}^{\prime}$ the lifetime which corresponds to $\left(\left(\alpha_{i}\right)_{i \in N \backslash S},\left(\alpha_{i}^{\prime}\right)_{i \in S}\right)$ and we adopt the convention $\frac{0}{0}=0$. We have:

$$
u_{i}\left(\left(x_{i}\right)_{i \in N \backslash S},\left(x_{i}^{\prime}\right)_{i \in S},\left(\alpha_{i}\right)_{i \in N \backslash S},\left(\alpha_{i}^{\prime}\right)_{i \in S}\right)=\frac{x_{i}^{\prime}}{\sum_{S \ni i} \alpha_{i}^{\prime}(S)}
$$


if $i \in S$ and

$$
u_{i}\left(\left(x_{i}\right)_{i \in N \backslash S},\left(x_{i}^{\prime}\right)_{i \in S},\left(\alpha_{i}\right)_{i \in N \backslash S},\left(\alpha_{i}^{\prime}\right)_{i \in S}\right)=\frac{x_{i}}{\sum_{S \ni i} \alpha_{i}(S)}=u_{i}\left(x,\left(\alpha_{i}\right)_{i \in N}\right)
$$

if $i \in N \backslash S$. The $\left(v,\left(\left(\alpha_{i}\right)_{i \in N \backslash S},\left(\alpha_{i}^{\prime}\right)_{i \in S}\right)\right)$-efficiency ensures that

$$
x^{\prime}(S)+x(N \backslash S) \leq \sum_{T \subseteq N} \bar{\alpha}^{\prime}(T) v(T) .
$$

Furthermore, $\left(\mathcal{A},\left(\lambda_{S}\right)_{S \subseteq N}\right)$ is a balanced system, then, $\forall i \in N, \sum_{S \ni i} \alpha_{i}(S) \leq \sum_{S \ni i} \lambda_{S}=1$, therefore, $\forall i \in N, u_{i}\left(x,\left(\alpha_{i}\right)_{i \in N}\right)=\frac{x_{i}}{\sum_{S \ni i} \alpha_{i}(S)} \geq x_{i}$. Since $x \in \mathrm{d}-\mathrm{C}(\tilde{v})$, we deduce that:

$$
\sum_{T \subseteq N} \bar{\alpha}^{\prime}(T) v(T) \leq \sum_{T \subseteq N} \alpha^{\prime}(T) x(T) \leq \sum_{T \subseteq N} \bar{\alpha}^{\prime}(T) \sum_{i \in T} u_{i}\left(x,\left(\alpha_{i}\right)_{i \in N}\right) .
$$

Then, we know that, $\forall i \in N, u_{i}\left(x,\left(\alpha_{i}\right)_{i \in N}\right) \leq u_{i}\left(\left(x_{i}\right)_{i \in N \backslash S},\left(x_{i}^{\prime}\right)_{i \in S},\left(\alpha_{i}\right)_{i \in N \backslash S},\left(\alpha_{i}^{\prime}\right)_{i \in S}\right)$ with equality for $i \in N \backslash S$ and strict inequality for $i \in S$. Hence,

$$
\sum_{T \subseteq N} \bar{\alpha}^{\prime}(T) \sum_{i \in T} u_{i}\left(x,\left(\alpha_{i}\right)_{i \in N}\right)<\sum_{T \subseteq N} \bar{\alpha}^{\prime}(T) \sum_{i \in T} u_{i}\left(\left(x_{i}\right)_{i \in N \backslash T},\left(x_{i}^{\prime}\right)_{i \in T},\left(\alpha_{i}\right)_{i \in N \backslash T},\left(\alpha_{i}^{\prime}\right)_{i \in T}\right) .
$$

A simple rewriting gives:

$$
\begin{gathered}
\sum_{T \subseteq N} \bar{\alpha}^{\prime}(T) \sum_{i \in T} u_{i}\left(\left(x_{i}\right)_{i \in N \backslash T},\left(x_{i}^{\prime}\right)_{i \in T},\left(\alpha_{i}\right)_{i \in N \backslash T},\left(\alpha_{i}^{\prime}\right)_{i \in T}\right)= \\
\sum_{i \in N} \sum_{T \ni i} \bar{\alpha}^{\prime}(T) u_{i}\left(\left(x_{i}\right)_{i \in N \backslash T},\left(x_{i}^{\prime}\right)_{i \in T},\left(\alpha_{i}\right)_{i \in N \backslash T},\left(\alpha_{i}^{\prime}\right)_{i \in T}\right) .
\end{gathered}
$$

The definition of $\bar{\alpha}^{\prime}$ ensures that $\forall T \subseteq N$, and $\forall i \in N, \bar{\alpha}^{\prime}(T) \leq \alpha_{i}(T)$. Hence,

$$
\begin{aligned}
\sum_{i \in N} \sum_{T \ni i} \bar{\alpha}^{\prime}(T) u_{i}\left(\left(x_{i}\right)_{i \in N \backslash T},\left(x_{i}^{\prime}\right)_{i \in T},\left(\alpha_{i}\right)_{i \in N \backslash T},\left(\alpha_{i}^{\prime}\right)_{i \in T}\right) \leq \\
\sum_{i \in S}\left(\frac{x_{i}^{\prime}}{\sum_{T \ni i} \bar{\alpha}^{\prime}(T)} \sum_{T \ni i} \bar{\alpha}^{\prime}(T)\right)+\sum_{i \in N \backslash S}\left(\frac{x_{i}}{\sum_{T \ni i} \bar{\alpha}^{\prime}(T)} \sum_{T \ni i} \bar{\alpha}^{\prime}(T)\right),
\end{aligned}
$$

and, finally,

$$
\sum_{i \in S}\left(\frac{x_{i}^{\prime}}{\sum_{T \ni i} \bar{\alpha}^{\prime}(T)} \sum_{T \ni i} \bar{\alpha}^{\prime}(T)\right)+\sum_{i \in N \backslash S}\left(\frac{x_{i}}{\sum_{T \ni i} \bar{\alpha}^{\prime}(T)} \sum_{T \ni i} \bar{\alpha}^{\prime}(T)\right)=x^{\prime}(S)+x(N \backslash S) .
$$

The inequalities (2) to (7) leads to the contradiction $x^{\prime}(S)+x(N \backslash S)<x^{\prime}(S)+x(N \backslash S)$.

We prove now the other statements of the theorem.

1 If $\exists i \in N$, such that $u_{i}\left(x,\left(\alpha_{j}\right)_{j \in N}\right)<0$ then the strategy $\left(x_{i}, \alpha_{i}\right)$ is strictly dominated by the strategy which consists in claiming a payoff $x_{i}^{\prime}=0$ for a strategic time allocation equals to 0 for every coalition.

2 We want to prove that $\forall S \subseteq N, \sum_{i \in S} u_{i}(x, \alpha) \geq v(S)$. Suppose there exists $S \subseteq N$ such that $\sum_{i \in S} u_{i}\left(x,\left(\alpha_{i}\right)_{i \in N}\right)<v(S)$. 
(a) If there exists $i \in N$ such that $u_{i}\left(x,\left(\alpha_{i}\right)_{i \in N}\right)=-1$ then it is clear that $\left(x,\left(\alpha_{i}\right)_{i \in N}\right)$ is not a strong Nash equilibrium.

(b) If $\forall i \in S, u_{i}\left(x,\left(\alpha_{i}\right)_{i \in N}\right) \neq-1$, the strategy of the players belonging to $S$ are strictly dominated by $\alpha_{i}^{\prime}(S)=\alpha_{i}(S)+1, \alpha_{i}^{\prime}(T)=\alpha_{i}(T)$ if $T \neq S, \forall i \in S$ and $x_{i}^{\prime}=x_{i}+$ $\frac{v(S)-\sum_{i \in S} u_{i}\left(x,\left(\alpha_{i}\right)_{i \in N}\right)}{|S|}$.

3 If $x(N)<G(v, \bar{\alpha})$ then there exists a deviation of the coalition $N$ which consists in claiming $x_{i}^{\prime}=x_{i}+\frac{G(v, \bar{\alpha})-x(N)}{|N|}$.

Observe that, unlike the d-core, the d-multicoalitional core has only nonnegative payoff vectors. We now show the converse result: the d-multicoalitional core is precisely the set of strategies satisfying requirements (i) to (iii).

Let $\left(x,\left(\alpha_{i}\right)_{i \in N}\right)$ be a couple satisfying (i), (ii) and (iii). We want to prove that the strategy $\left(x,\left(\alpha_{i}\right)_{i \in N}\right)$ belongs to the d-multicoalitional core. Suppose there exists for a coalition $S \subseteq N$ a coalitional deviation $\left(\left(x_{i}^{\prime}\right)_{i \in S},\left(\alpha_{i}^{\prime}\right)_{i \in S}\right)$ such that

$$
\forall i \in S, u_{i}\left(x,\left(\alpha_{i}\right)_{i \in N}\right)<u_{i}\left(\left(x_{i}\right)_{i \in N \backslash S},\left(x_{i}^{\prime}\right)_{i \in S},\left(\alpha_{i}\right)_{i \in N \backslash S},\left(\alpha_{i}^{\prime}\right)_{i \in S}\right) .
$$

Since $\forall i \in N, 0 \leq u_{i}\left(x,\left(\alpha_{i}\right)_{i \in N}\right)$, the strict inequality

$$
\forall i \in S, u_{i}\left(x,\left(\alpha_{i}\right)_{i \in N}\right)<u_{i}\left(\left(x_{i}\right)_{i \in N \backslash S},\left(x_{i}^{\prime}\right)_{i \in S},\left(\alpha_{i}\right)_{i \in N \backslash S},\left(\alpha_{i}^{\prime}\right)_{i \in S}\right)
$$

and the definition of $u_{i}$ ensure that the $\left(v,\left(\left(\alpha_{i}\right)_{i \in N \backslash S},\left(\alpha_{i}^{\prime}\right)_{i \in S}\right)\right)$-efficiency is satisfied. We denote by $\bar{\alpha}^{\prime}$ the lifetime which corresponds to $\left(\left(\alpha_{i}\right)_{i \in N \backslash S},\left(\alpha_{i}^{\prime}\right)_{i \in S}\right)$ and we adopt the convention $\frac{0}{0}=0$. Since $u_{i}\left(x,\left(\alpha_{i}\right)_{i \in N}\right) \geq 0$, we have:

$$
u_{i}\left(\left(x_{i}\right)_{i \in N \backslash S},\left(x_{i}^{\prime}\right)_{i \in S},\left(\alpha_{i}\right)_{i \in N \backslash S},\left(\alpha_{i}^{\prime}\right)_{i \in S}\right)=\frac{x_{i}^{\prime}}{\sum_{S \ni i} \alpha_{i}^{\prime}(S)}
$$

if $i \in S$ and

$$
u_{i}\left(\left(x_{i}\right)_{i \in N \backslash S},\left(x_{i}^{\prime}\right)_{i \in S},\left(\alpha_{i}\right)_{i \in N \backslash S},\left(\alpha_{i}^{\prime}\right)_{i \in S}\right)=\frac{x_{i}}{\sum_{S \ni i} \alpha_{i}(S)}=u_{i}\left(x,\left(\alpha_{i}\right)_{i \in N}\right)
$$

if $i \in N \backslash S$. The $\left(v,\left(\left(\alpha_{i}\right)_{i \in N \backslash S},\left(\alpha_{i}^{\prime}\right)_{i \in S}\right)\right)$-efficiency ensures that

$$
x^{\prime}(S)+x(N \backslash S) \leq \sum_{T \subseteq N} \bar{\alpha}^{\prime}(T) v(T) .
$$

Furthermore, by hypothesis,

$$
\sum_{T \subseteq N} \bar{\alpha}^{\prime}(T) v(T) \leq \sum_{T \subseteq N} \bar{\alpha}^{\prime}(T) \sum_{i \in T} u_{i}\left(x,\left(\alpha_{i}\right)_{i \in N}\right) .
$$

Then, we deduce

$$
\sum_{T \subseteq N} \bar{\alpha}^{\prime}(T) \sum_{i \in T} u_{i}\left(x,\left(\alpha_{i}\right)_{i \in N}\right)<x^{\prime}(S)+x(N \backslash S),
$$

a contradiction.

Proposition 3. Let $\left(x,\left(\alpha_{i}\right)_{i \in N}\right)$ be an element of the d-multicoalitional core, and $i \in N$ such that $u_{i}\left(x,\left(\alpha_{i}\right)_{i \in N}\right) \neq 0$. Then for all $S \ni i$, we have $\alpha_{i}(S)=\bar{\alpha}(S)$. 
Proof. By definition of $u_{i}$, we have $u_{i}=\frac{x_{i}}{\sum_{S \ni i} \alpha_{i}(S)}$. Since $\sum_{S \ni i} \alpha_{i}(S) \geq \sum_{S \ni i} \bar{\alpha}(S)$, choosing $\alpha_{i} \neq \bar{\alpha}$ would make the strategy $\left(x,\left(\alpha_{i}\right)_{i \in N}\right)$ dominated.

Essentially, the proposition says that in an equilibrium, all players ask for the same time allocation $\bar{\alpha}$. The important consequence of this is, if all players can reach a positive income, then the d-multicoalitional core is a multicoalitional solution.

Proposition 4. Let $\left(x,\left(\alpha_{i}\right)_{i \in N}\right)$ an element of the d-multicoalitional core of a game $v$. Let $S$ be a coalition such that $\bar{\alpha}(S)>0$. Then $\sum_{i \in S} u_{i}\left(x,\left(\alpha_{i}\right)_{i \in N}\right)=v(S)$.

Proof. Let $S$ be a coalition such that $\bar{\alpha}(S)>0$. Then if $\sum_{i \in S} \frac{x(i)}{\sum_{S \ni i} \alpha_{i}(S)}>v(S)$, we have by Theorem 3

$$
x(N)=\sum_{T \subseteq N} \bar{\alpha}(T) v(T)<\sum_{T \subseteq N} \bar{\alpha}(T) \sum_{i \in T} u_{i}\left(x,\left(\alpha_{i}\right)_{i \in N}\right)=\sum_{i \in N} \sum_{T \ni i} \bar{\alpha}(T) \frac{x_{i}}{\sum_{T \ni i} \bar{\alpha}(T)}=x(N),
$$

a contradiction.

Then $\alpha(S)>0$ implies $\sum_{i \in S} u_{i}\left(x,\left(\alpha_{i}\right)_{i \in S}\right)=v(S)$.

Remark 2. The assumptions of the model come down to consider that players receive their payoff at the end of the coalition formation process. Hence, according to the fact that players want to maximize their average "hourly payoff", the distribution of coalitional payoffs (worth) is not performed at each point of time but at the end of the period of activity. In our model, it is not important for players to know at each instant of time how the coalitions share the worth. For providing a comprehensive picture, a worker wants to know how much is her pay at the end of each month comparatively to the number of working hours, and is not interested in how the company divided her worth at each instant of time. However it is clear, according to Proposition 4, that a solution given by the d-multicoalitional core is, in particular, a possible and natural rational sharing of worth in each point of time activity.

Proposition 5. If there exists $\left(x,\left(\alpha_{i}\right)_{i \in N}\right)$ such that $\left.\left(u_{i}\left(x,\left(\alpha_{i}\right)_{i \in N}\right)\right)\right)_{i \in N} \in \mathrm{d}-\mathrm{C}(v)$ and $u_{i}\left(x,\left(\alpha_{i}\right)_{i \in N}\right) \geq$ $0 \forall i \in N$, then $\left(x,\left(\alpha_{i}\right)_{i \in N}\right) \in \mathrm{d}-M C(v)$.

Proof. The proof is similar to that of Theorem 3.

Remark 3. As we can see from the following example, unlike the d-core or the $\mathcal{B}$-core (Cesco, 2012 ) it is not necessary to require that each player is active during exactly one unit of time. Moreover, $\left(x,\left(\alpha_{i}\right)_{i \in N}\right)$ in the d-multicoalitional core does not necessarily imply that $\left.\left(u_{i}\left(x,\left(\alpha_{i}\right)_{i \in N}\right)\right)\right)$ is in the d-core.

Consider the game $v$ defined over the subset of $N=\{1,2,3\}$ by:

\begin{tabular}{|c|ccccccc|}
\hline$S$ & 1 & 2 & 3 & 12 & 13 & 23 & 123 \\
\hline$v(S)$ & 1 & 1 & 1 & 3 & 3 & 3 & 4 \\
\hline
\end{tabular}

and the strategy $\left(x,\left(\alpha_{i}\right)_{i \in N}\right)$ defined by: $x(1)=3, x(2)=x(3)=2$ and $\alpha_{1}(1)=\alpha_{1}(12)=$ $\alpha_{1}(13)=1, \alpha_{2}(12)=\alpha_{3}(13)=1$, and $\alpha_{i}(S)=0$ otherwise. This yields

$$
x(N)=7=\sum_{S \subseteq N} \bar{\alpha}(S) v(S), u_{1}\left(x,\left(\alpha_{i}\right)_{i \in N}\right)=1, u_{2}\left(x,\left(\alpha_{i}\right)_{i \in N}\right)=u_{3}\left(x,\left(\alpha_{i}\right)_{i \in N}\right)=2 .
$$

Then $\left(x,\left(\alpha_{i}\right)_{i \in N}\right) \in \mathrm{d}-M C(v)$ but $\left(u_{i}\left(x,\left(\alpha_{i}\right)_{i \in N}\right)\right)_{i \in N} \notin \mathrm{d}-\mathrm{C}(v)$, because $\sum_{i \in N} u_{i}\left(x,\left(\alpha_{i}\right)_{i \in N}\right)=$ $5>4.5=v(N)+\bar{t}(v)$.

The following proposition gives a result very close to the one of (Bejan and Gómez, 2012a). 
Proposition 6. If $\left(x,\left(\alpha_{i}\right)_{i \in N}\right) \in \mathbb{R}^{N} \times \prod_{j \in N} \mathcal{T}_{j}$ is such that $\bar{\alpha}$ is in standard form, and $x_{i} \geq 0$ for all $i \in N$, then the following equivalence is satisfied:

$$
\left(x,\left(\alpha_{i}\right)_{i \in N}\right) \in \mathrm{d}-M C(v) \Leftrightarrow\left(u_{i}\left(x,\left(\alpha_{i}\right)_{i \in N}\right)\right)_{i \in N} \in \mathrm{d}-\mathrm{C}(v)
$$

Proof. By Proposition 5, it suffices to prove the right implication. Let $\left(x,\left(\alpha_{i}\right)_{i \in N}\right) \in \mathrm{d}-M C(v)$, we have $\sum_{i \in S} u_{i}\left(x,\left(\alpha_{i}\right)_{i \in N}\right) \geq v(S)$ for every $S \subseteq N$. Hence,

$$
v(N)+\bar{t}(v) \leq \sum_{i \in N} u_{i}\left(x,\left(\alpha_{i}\right)_{i \in N}\right) .
$$

Furthermore, by Proposition 4, we have equality if $\bar{\alpha}(S)>0$. Hence, since $\bar{\alpha}$ is in standard form, there exists $\left(\delta_{S}\right)_{S \in\{T \subseteq N, \bar{\alpha}(T)>0\}}$ such that

$$
\sum_{i \in N} u_{i}\left(x,\left(\alpha_{i}\right)_{i \in N}\right)=\sum_{S \in\{T \subseteq N, \bar{\alpha}(T)>0\}} \delta_{S} \sum_{i \in S} u_{i}\left(x,\left(\alpha_{i}\right)_{i \in N}\right)=\sum_{S \in\{T \subseteq N, \bar{\alpha}(T)>0\}} \delta_{S} v(S) \leq v(N)+\bar{t}(v) .
$$

By combining with (8), equality holds throughout, and $\left(u_{i}\left(x,\left(\alpha_{i}\right)_{i \in N}\right)\right)_{i \in N} \in \mathrm{d}-\mathrm{C}(v)$.

\section{A motivating example}

The following example is inspired by the simple example coming from Osborne and Rubinstein (1994)[p.259] of a production economy where a capitalist owns a factory and each of the workers owns only his own labour power, and they can produce something only together with the capitalist. Observe that, by Theorem 3, a coalition with a negative value cannot be formed; this observation leads to easily generalize the results of the d-multicoalitional core to cooperative games with restricted coalitions. Indeed, it suffices to allocate a negative worth to unfeasible coalitions. In our example, we will suppose for simplicity that we have two capitalists (owners of a physical capital) denoted by 1 and 2, and three workers denoted by 3,4 and 5 . We assume that a coalition produces a nonnegative output if and only if it contains at least one worker and one capitalist. The daily output of a coalition $S$ is the integer part of a Cobb-Douglas function:

$$
v(S)=10 \times\left\lfloor(\text { number of capitalists in } S)^{1 / 2} \times(\text { number of workers in } S)^{1 / 3}\right\rfloor,
$$

and

$$
v(S)=-1 \text { if } S \text { is not feasible }
$$

Hence

$$
\begin{aligned}
& v(\text { one investor, one worker })=10 \\
& v(\text { two investors, one worker })=14 \\
& v(\text { one investor, two workers })=12 \\
& v(\text { two investors, two workers })=17 \\
& v \text { (one investor, three workers })=14 \\
& v(\text { two investors, three workers })=20
\end{aligned}
$$

Clearly the core of $v$ is empty. However, if each of the coalitions 13, 14, 15, 23, 24, 25 is active for half a day, the coalitions can produce 30 in total. A corresponding strategy is: 
(i) each capitalist is active during half a day with each worker and claims 7.5

(ii) each worker is active during half a day with each capitalist and claims 5

This strategy is an element of the d-multicoalitional core because the daily outcome is equal to 5 for every agent, which is efficient, positive and coalitionally rational.

The corresponding time allocation $\alpha$ is

\begin{tabular}{|c|cccccc|}
\hline$S$ & 13 & 14 & 15 & 23 & 24 & 25 \\
\hline$\alpha(S)$ & $1 / 2$ & $1 / 2$ & $1 / 2$ & $1 / 2$ & $1 / 2$ & $1 / 2$ \\
\hline
\end{tabular}

and a possible optimal timetable is given on Figure 2 .

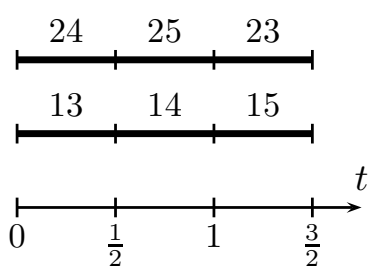

Figure 2: A possible optimal timetable

The minimal duration of the coalition formation process is $d(\alpha)=\frac{3}{2}$. Player 1 receives 5 per "hour lived", and player 3 receives $\frac{5}{\frac{3}{2}}=\frac{10}{3}$ per "hour lived". However, according to (9), coalition 13 could produce 15 together in $d(\alpha)=\frac{3}{2}$ unit of time. This production of 15 could be shared as follows:

- 1 receives 8. This amount corresponds to $\frac{8}{\frac{3}{2}}=\frac{16}{3}$ per hour lived instead of 5

- 3 receives 7 . This amount corresponds to $\frac{7}{\frac{3}{2}}=\frac{14}{3}$ per hour lived instead of $\frac{10}{3}$.

With this sharing, players 1 and 3 received strictly more according to the utility per total unit of time. Hence, there exists a profitable deviation of coalition 13 from the point of view of the utility $\tilde{u}_{i}$.

Now, consider the strategy which leads to the situation where each of the coalitions 13 and 245 is active for one day:

The corresponding time allocation $\alpha$ is

\begin{tabular}{|c|cc|}
\hline$S$ & 13 & 245 \\
\hline$\alpha(S)$ & 1 & 1 \\
\hline
\end{tabular}

and the unique optimal timetable is given on Figure 3.

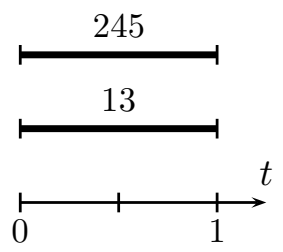

Figure 3: The unique optimal timetable 
The coalitions can produce 22 in total in one day, a possible strategic payoff is $x_{1}=x_{2}=8$ and $x_{3}=x_{4}=x_{5}=2$. This strategy is an element of the d-multicoalitional core. Furthermore, the minimal duration of the coalition formation process is $d(\alpha)=1$. This payoff is also an element of the d-core and even of the c-core, a fact which illustrates Proposition 6. In addition, the reader can check that there is no possible coalitional deviation according to the utility $\tilde{u}$. Hence, this strategy seems more appealing since it is stable for $u$ as well as for $\tilde{u}$.

We are going to examine in the next section the selections of strong Nash equilibria of our game which are strong Nash equilibria according to the utilities $u_{i}$ and $\tilde{u}_{i}$.

\section{The c-multicoalitional core}

Let $v$ be a TU-game, we denote by $S N \tilde{u}(v)$ the set of strong Nash equilibria of $\left(N ; \mathbb{R} \times \mathcal{T}_{1}, \cdots, \mathbb{R} \times\right.$ $\left.\mathcal{T}_{n} ; \tilde{u}_{1}, \cdots, \tilde{u}_{n}\right)$. The next proposition shows that these strong Nash equilibria have properties similar to elements of the d-multicoalitional core.

Proposition 7. For any $\left(x,\left(\alpha_{i}\right)_{i \in N}\right) \in S N \tilde{u}(v)$, we have:

(i) $\forall i \in N, \tilde{u}_{i}\left(x,\left(\alpha_{i}\right)_{i \in N}\right) \geq 0$

(ii) $\forall S \subseteq N, \sum_{i \in S} \tilde{u}_{i}\left(x,\left(\alpha_{i}\right)_{i \in N}\right) \geq v(S)$ (coalitional rationality)

(iii) $x(N)=G(v, \bar{\alpha})((v, \bar{\alpha})$-efficiency $)$

Proof. The proof is similar to the corresponding points of Theorem 3.

The next proposition investigates the nonemptiness of the set of such equilibria.

Proposition 8. $S N \tilde{u}(v) \neq \emptyset$ if and only if $S N \tilde{u}(v) \cap \mathrm{d}-M C(v) \neq \emptyset$.

Proof. (i) Suppose $S N \tilde{u}(v) \neq \emptyset$, and let $\left(x,\left(\alpha_{i}\right)_{i \in N}\right)$ be an element of $S N \tilde{u}(v)$. By using Theorem 2, we know that:

$$
\max _{\mathcal{C}: \text { clique of } G} \alpha(\mathcal{C}) \leq d(\alpha) \text {. }
$$

Hence, by using the convention $\frac{0}{0}=0$ and since $\forall i \in N, x_{i} \geq 0$, and $\{S \ni i, \bar{\alpha}(S)>0\}$ is a clique, we have:

$$
\frac{x_{i}}{d(\bar{\alpha})} \leq \frac{x_{i}}{\sum_{S \ni i} \bar{\alpha}(S)}
$$

We put $\bar{\alpha}_{i}(S)=\bar{\alpha}(S)$ for all $i \in N$ and $S \subseteq N$. We deduce that:

$$
\tilde{u}_{i}\left(x,\left(\alpha_{i}\right)_{i \in N}\right) \leq u_{i}\left(x,\left(\bar{\alpha}_{i}\right)_{i \in N}\right) .
$$

Hence, if $x_{i}=0$, we have $\tilde{u}_{i}\left(x,\left(\alpha_{i}\right)_{i \in N}\right)=u_{i}\left(x,\left(\bar{\alpha}_{i}\right)_{i \in N}\right)$ and if $x_{i}>0$, there exists an active coalition $S$ under $\bar{\alpha}$ which contains $i$, and by using Propositions 3 and 7 ,

$$
v(S) \leq \sum_{i \in S} \tilde{u}_{i}\left(x,\left(\alpha_{i}\right)_{i \in N}\right) \leq \sum_{i \in S} u_{i}\left(x,\left(\bar{\alpha}_{i}\right)_{i \in N}\right)=v(S)
$$

from which we deduce the equality

$$
\tilde{u}_{i}\left(x,\left(\alpha_{i}\right)_{i \in N}\right)=u_{i}\left(x,\left(\bar{\alpha}_{i}\right)_{i \in N}\right) \quad \forall i \in N .
$$

It is clear that:

$$
\tilde{u}_{i}\left(x,\left(\alpha_{i}\right)_{i \in N}\right)=\tilde{u}_{i}\left(x,\left(\bar{\alpha}_{i}\right)_{i \in N}\right) \quad \forall i \in N .
$$

Therefore $\left(x,\left(\bar{\alpha}_{i}\right)_{i \in N}\right) \in S N \tilde{u}(v)$ and furthermore, we have by Proposition 7: 
(a) $u_{i}\left(x,\left(\bar{\alpha}_{i}\right)_{i \in N}\right) \geq 0$.

(b) $\forall S \subseteq N, \sum_{i \in S} u_{i}\left(x,\left(\bar{\alpha}_{i}\right)_{i \in N}\right) \geq v(S)$.

(c) $x(N)=\sum_{S \subseteq N} \bar{\alpha}(S) v(S)$.

Therefore $\left(x,\left(\bar{\alpha}_{i}\right)_{i \in N}\right) \in S N \tilde{u}(v) \cap \mathrm{d}-M C(v)$

(ii) The converse is obvious.

Unlike the case where the utility is defined per active unit of time, it could be the case that no strong equilibrium exists when utility per total unit of time is used instead. The foregoing proposition has shown that if a strong Nash equilibrium exists, it can be turned into a strong equilibrium of both problems, i.e., with the two types of utility. The following definition is a natural consequence of this fact: let $v$ be a TU-game, we denote by $c-M C(v)$ and we call c-multicoalitional core of $v$ the set

$$
\mathrm{c}-M C(v):=S N \tilde{u}(v) \cap \mathrm{d}-M C(v) .
$$

Elements of the c-multicoalitional core have the remarkable property to be undominated strategies both for utility per active unit of time and total unit of time. Since the c-multicoalitional core is a subset of the d-multicoalitional core, it follows that it is also a multicoalitional solution. The next theorem gives a necessary and sufficient condition for the nonemptiness of the c-multicoalitional core. It turns out that this condition is equivalent to the nonemptiness of the c-core of a related game.

Theorem 4. Let $v \in \mathcal{G}(N)$ be a game, and denote by $\tilde{v}$ the game defined by $\tilde{v}(i)=0$ if $v(i)<0$ and $\tilde{v}(S)=v(S)$ otherwise. Then c- $M C(v) \neq \emptyset$ if and only if $\tilde{v}$ is c-balanced, and moreover

$$
\mathrm{c}-\mathrm{C}(\tilde{v})=\left\{\left(\tilde{u}_{i}\left(x,\left(\alpha_{i}\right)_{i \in N}\right)\right)_{i \in N} \mid\left(x,\left(\alpha_{i}\right)_{i \in N}\right) \in \mathrm{c}-M C(v)\right\} .
$$

Proof. (i) Let us prove that if the c-multicoalitional core is nonempty, then so is the c-core. Let us take $\left(x,\left(\alpha_{i}\right)_{i \in N}\right) \in \mathrm{c}-M C(v)$ and prove that $\left(\tilde{u}_{i}\left(x,\left(\alpha_{i}\right)_{i \in N}\right)\right)_{i \in N} \in \mathrm{c}-\mathrm{C}(\tilde{v})$. Firstly, we have

$$
x(N)=\sum_{S \subseteq N} \bar{\alpha}(S) v(S) \leq \sum_{S \subseteq N} \bar{\alpha}(S) \tilde{v}(S) .
$$

Moreover, since $\tilde{u}_{i}\left(x,\left(\alpha_{i}\right)_{i \in N}\right) \geq 0$ and $\sum_{i \in S} \tilde{u}_{i}\left(x,\left(\alpha_{i}\right)_{i \in N}\right) \geq v(S)$, we deduce easily that $\sum_{i \in S} \tilde{u}_{i}\left(x,\left(\alpha_{i}\right)_{i \in N}\right) \geq \tilde{v}(S)$. This establishes coalitional rationality. In addition, we can deduce that

$$
\sum_{S \subseteq N} \bar{\alpha}(S) \tilde{v}(S) \leq \sum_{S \subseteq N} \bar{\alpha}(S) \sum_{i \in S} \tilde{u}_{i}\left(x,\left(\alpha_{i}\right)_{i \in N}\right) \leq \sum_{i \in N} x_{i} \sum_{S \ni i} \frac{\bar{\alpha}(S)}{d(\bar{\alpha})}
$$

According to Theorem $2, \sum_{S \ni i} \bar{\alpha}(S) \leq d(\bar{\alpha})$, therefore

$$
\sum_{i \in N} x_{i} \sum_{S \ni i} \frac{\bar{\alpha}(S)}{d(\bar{\alpha})} \leq x(N) .
$$

Hence, equality holds throughout and for $i \in N$, if $x_{i}>0$ then $\sum_{S \ni i} \bar{\alpha}(S)=d(\bar{\alpha})$, while if $x_{i}=0$ then $\tilde{v}(i)=0$ and $\sum_{S \ni i} \bar{\alpha}(S) \leq d(\bar{\alpha})$.

We put:

$$
\alpha_{i}^{\prime}(i)=\alpha_{i}(i)+\left(d(\bar{\alpha})-\sum_{S \ni i} \bar{\alpha}(S)\right), \quad \text { if } x_{i}=0
$$


and

$$
\alpha_{i}^{\prime}(S)=\alpha_{i}(S), \quad \text { otherwise. }
$$

Then we have $d\left(\bar{\alpha}^{\prime}\right)=d(\bar{\alpha})=\sum_{S \ni i} \bar{\alpha}_{i}^{\prime}(S)$. Let $f_{\bar{\alpha}^{\prime}}$ be an optimal timetable of $\bar{\alpha}^{\prime}$. It is clear that $\forall t \in\left[0, d\left(\bar{\alpha}^{\prime}\right)\right], f_{\bar{\alpha}^{\prime}}(t) \in \Pi(N)$.

Since $\Pi(N)$ is a finite set, we deduce that there exists $\pi_{1}, \ldots, \pi_{k} \in \Pi(N)$ and $p_{1}, \ldots, p_{k} \in$ $\left[0, d\left(\bar{\alpha}^{\prime}\right)\right]$ such that

$$
\forall j \in\{1, \ldots, k\}, \quad \lambda\left(\left\{t \in\left[0, d\left(\bar{\alpha}^{\prime}\right)\right], f_{\bar{\alpha}^{\prime}}(t)=\pi_{j}\right\}\right)=p_{j},
$$

where $\lambda$ is the Lebesgue measure, and $\sum_{j=1}^{k} p_{j}=d\left(\bar{\alpha}^{\prime}\right)$. The time consistency given by the definition of the timetable implies $\lambda\left(\left\{t \in\left[0, d\left(\bar{\alpha}^{\prime}\right)\right], S \in f_{\bar{\alpha}^{\prime}}(t)\right\}\right)=\bar{\alpha}^{\prime}(S)$ for every $S \subseteq N$, we have $\sum_{j \mid \pi_{j} \ni S} p_{j}=\bar{\alpha}^{\prime}(S)$ for every $S \subseteq N$, hence

$$
\sum_{j=1}^{k} p_{j}\left(\sum_{S \in \pi_{j}} \tilde{v}(S)\right)=\sum_{S \subseteq N} \bar{\alpha}^{\prime}(S) \tilde{v}(S)
$$

Furthermore

$$
\sum_{j=1}^{k} p_{j}\left(\sum_{S \in \pi_{j}} \tilde{v}(S)\right) \leq d(\bar{\alpha})\left(\max _{\pi \in \Pi(N)} \sum_{S \in \pi} v(S)\right) \leq d(\bar{\alpha})(\tilde{v}(N)+\bar{t}(\tilde{v})) .
$$

Therefore, we deduce that $x(N) \leq d(\bar{\alpha})(\tilde{v}(N)+\bar{t}(\tilde{v}))$. Since, $\tilde{u}_{i}\left(x,\left(\alpha_{i}\right)_{i \in N}\right) \geq 0 \forall i \in N$ and $\sum_{i \in S} \tilde{u}_{i}\left(x,\left(\alpha_{i}\right)_{i \in N}\right) \geq \tilde{v}(S) \forall S \subseteq N$, the definition of $\tilde{u}_{i}$ and $\bar{t}(\tilde{v})$ gives $x(N) \geq$ $d(\bar{\alpha})(\tilde{v}(N)+\bar{t}(\tilde{v}))$.

and finally,

$$
x(N)=d(\bar{\alpha})\left(\max _{\pi \in \Pi(N)} \sum_{S \in \pi} \tilde{v}(S)\right)=d(\bar{\alpha})(\tilde{v}(N)+\bar{t}(\tilde{v})) .
$$

Hence, $\left(\frac{x_{i}}{d(\bar{\alpha})}\right)_{i \in N}=\left(\tilde{u}_{i}\left(x,\left(\alpha_{i}\right)_{i \in N}\right)\right)_{i \in N} \in \mathrm{c}-\mathrm{C}(\tilde{v})$.

(ii) We prove the converse implication. If $\tilde{v}$ is c-balanced, then there exists a partition $\pi \in$ $\Pi(N)$ such that $\sum_{S \in \pi} \tilde{v}(S)=\tilde{v}(N)+\bar{t}(v)$. Let $x \in \mathrm{c}-\mathrm{C}(\tilde{v})$. It is easy to verify that $\left(x,\left(\alpha_{i}\right)_{i \in N}\right)$, with $\alpha_{i}(S)=1$ if $(i \in S$ and $S \in \pi)$ and $\alpha_{i}(S)=0$ otherwise, belongs to the c-multicoalitional core.

Remark 4. There exist games which are not c-balanced, but with a nonempty c-multicoalitional core. For example, the game $v$ defined on $N=\{1,2,3\}$ by

\begin{tabular}{|c|ccccccc|}
\hline$S$ & 1 & 2 & 3 & 12 & 13 & 23 & 123 \\
\hline$v(S)$ & 0 & 0 & -100 & 10 & 0 & 0 & 0 \\
\hline
\end{tabular}

is not c-balanced. However, it can be checked that $\left(x,\left(\alpha_{i}\right)_{i \in N}\right)$ with $x=(5,5,0)$ and $\alpha_{1}(12)=$ $\alpha_{2}(12)=1, \alpha_{i}(S)=0$ otherwise, is an element of c-MC $(v)^{8}$.

\footnotetext{
${ }^{8}$ We observe, in particular, that it is more rational in our framework that any agent with a nonpositive productivity does not work, while most of classical models require that this kind of agents work during exactly one unit of time.
} 


\section{References}

W. Albers. Zwei Lösungskonzepte für kooperative Mehrpersonenspiele, die auf Anspruchsnivaus der Spieler basieren. OR-Verfahren/ Methods of Operations Research, XVIII:1-8, 1974.

W. Albers. Core- and kernel-variants based on imputations and demand profiles. In O. Moeschlin and D. Pallaschke, editors, Game Theory and Related Topics. North-Holland, Amsterdam, 1979 .

R. J. Aumann. Acceptable points in general cooperative $n$-person games. In Contributions to the Theory of Games IV, pages 287-324. Princeton University Press, Princeton, 1959.

R.J. Aumann. Lectures on Game Theory. Underground Classics in Economics. Westview Press, Amsterdam, 1989.

C. Bejan and J. C. Gómez. Using the aspiration core to predict coalition formation. International Game Theory Review, 2012a.

C. Bejan and J. C. Gómez. Axiomatizing core extensions. International Journal of Game Theory, 41(4):885-898, 2012b.

E. Bennett. The aspiration approach to predicting coalition formation and payoff distribution in sidepayment games. International Journal of Game Theory, 12(1):1-28, 1983.

O. Bondareva. Some applications of linear programming methods to the therory of cooperative games. Problemy Kibernetiki, 10:119-139, 1963.

J. C. Cesco. Nonempty core-type solutions over balanced coalitions in tu-games. International Game Theory Review, 14(03), 2012.

J. Cross. Some theoretic characteristics of economic and political coalitions. Journal of Conflict Resolution, 11:184-195, 1967.

S.D. Flam and L. Koutsougeras. Private information, transferable utility, and the core. Economic Theory, 42:591-609, 2010.

R. Garratt and C. Z Qin. On market games when agents cannot be in two places at once. Games and Economic Behavior, 31(2):165-173, 2000.

D. Gillies. Some theorems on n-person games. PhD thesis, Princeton, New Jersey, 1953.

D. Gillies. Solutions to general non-zero-sum games. Contributions to the Theory of Games, 4: 47-85, 1959.

S. Gonzalez and M. Grabisch. Preserving coalitional rationality for non-balanced games. International Journal of Game Theory, 44(3):733-760, 2015.

M. Grabisch and P. Miranda. On the vertices of the $k$-additive core. Discrete Mathematics, 308:5204-5217, 2008.

R. Guesnerie and C. Oddou. On economic games which are not necessarily superadditive:solution concept and application to a local public good problem with few a agents. Economics Letters, 3:301-306, 1979.

Y. Kannai. The core and balancedness. In R. J Aumann and S. Hart, editors, Handbook of Game Theory with Economic Applications. North-Holland, Amsterdam, 1992. 
L. Á. Kóczy and L. Lauwers. The coalition structure core is accessible. Games and Economic Behavior, 48:86-93, 2004.

M. J. Osborne and A. Rubinstein. A course in game theory. MIT press, 1994.

B. Peleg and P. Sudhölter. Introduction to the theory of cooperative games. Kluwer Academic Publisher, 2003.

L. S. Shapley. On balanced sets and cores. Naval Research Logistics Quaterly, 14:453-460, 1967.

B. Shitovitz. A comparison between the core and the monopoly solutions in a mixed exchange economy. Economic Theory, 10:559-563, 1997.

N. Sun, W. Trockel, and Z. Yang. Competitive outcomes and endogenous coalition formation in an n-person game. Journal of Mathematical Economics, 44:853-860, 2008.

W. Trockel. Core-equivalence for the nash bargaining solution. Economic Theory, 25:255-263, 2005.

G. Turbay. On value theories for n-person cooperative games. PhD thesis, Rice univ, Houston Tx, 1977. 